\title{
HYDROMAGNETIC FLOW AND HEAT TRANSFER BETWEEN TWO HORIZONTAL PLATES, THE LOWER PLATE BEING A STRETCHING SHEET*
}

\author{
BY
}

\author{
A. K. BORKAKOTI AND A. BHARALI
}

Dibrugarh University, Dibrugarh, Assam

Summary. Hydromagnetic flow and heat transfer between two horizontal parallel plates, where the lower one is a stretching sheet and the upper one is a porous solid plate are studied in the presence of a transverse magnetic field. Fluid velocities and temperature distributions are obtained and plotted graphically.

1. Introduction. Srivastava [1] has discussed the flow of second-order fluids with heat transfer between two plates, one moving and the other at rest. Srivastava and Sharma [2] have studied the same problem in a Newtonian electrically conducting fluid in the presence of a transverse magnetic field. Borkakati and Bharali [3] have discussed the heat transfer in the MHD flow between two coaxial non-conducting porous disks when one rotates and the other is at rest [4]. Recently Gupta and Chakraborti [5] have investigated the motion of an electrically conducting fluid past a horizontal plate in presence of a magnetic field, the motion being caused solely by the stretching of the plate.

The study to be reported herein, which is an extension of [5], considers the flow and heat transfer in the presence of another stationary porous plate through which there is a uniform injection. Fluid motion is due to the stretching of the plate and the injection through the upper solid porous plate. It is observed that when the injection velocity and the magnetic field are constant, the temperature of the fluid decreases with the increase of the stretching force of the lower plate.

2. Fluid motion. The flow of an incompressible viscous electrically conducting fluid between two horizontal parallel non-conducting plates is considered in the presence of a transverse magnetic field. A cartesian coordinate system is used where the $y$-axis is perpendicular to the plates located at $y=+h$ and $y=-h$. The lower plate is stretched by introducing two equal and opposite forces so that the position of the point $(0,-h)$ remains unchanged. The fluid is injected through the upper solid porous plate with constant velocity $V_{0}$. The induced magnetic field is neglected, which is valid for small magnetic Reynolds number; the external electric field is zero and the electric field due to polarization of charges is negligible [5]. Under the above assumptions, the equations governing

* Received May 29, 1981; revised version received January 19, 1982. The authors are grateful to the referee for his valuable suggestions. 
the steady flow are

$$
\begin{gathered}
u \frac{\partial u}{\partial x}+v \frac{\partial u}{\partial y}=-\frac{1}{\rho} \frac{\partial p}{\partial x}+v\left[\frac{\partial^{2} u}{\partial x^{2}}+\frac{\partial^{2} u}{\partial y^{2}}\right]-\frac{\sigma B_{0}^{2}}{\rho} u \\
u \frac{\partial v}{\partial x}+v \frac{\partial v}{\partial y}=-\frac{1}{\rho} \frac{\partial p}{\partial y}+v\left[\frac{\partial^{2} v}{\partial x^{2}}+\frac{\partial^{2} v}{\partial y^{2}}\right] \\
\frac{\partial u}{\partial x}+\frac{\partial v}{\partial y}=0
\end{gathered}
$$

where $u, v$ are the fluid velocity components along the $x$ and $y$ directions. All variables are assumed to be independent of $z$.

The boundary conditions are:

$$
u=c x, \quad v=0 \quad \text { at } y=-h ; \quad u=0, \quad v=-v_{0} \quad \text { at } y=+h
$$

We assume

$$
u=c x f^{\prime}(\eta), \quad v=-\operatorname{chf}(\eta), \quad \eta=y / h
$$

where a prime denotes differentiation with respect to $\eta$ and $u=c x$ represents the velocity of the lower plate with $c>0$. Substituting (5) into Eqs. (1) and (2), we have

$$
\begin{aligned}
-\frac{1}{\rho} \frac{\partial p}{\partial x} & =c^{2} x\left[f^{\prime 2}-f f^{\prime \prime}-\frac{1}{R} f^{\prime \prime \prime}+\frac{M^{2}}{R} f^{\prime}\right], \\
& -\frac{1}{\rho \eta} \frac{\partial p}{\partial \eta}=c^{2} h\left[f f^{\prime}+\frac{1}{R} f^{\prime \prime}\right],
\end{aligned}
$$

where

$$
R=\left(c h^{2} / v\right), \quad M=(\sigma / \rho v)^{1 / 2} B_{0} h .
$$

Differentiating (7) with respect to $x$, we have $\partial^{2} p / \partial x \partial \eta=0$, which suggests that we consider

$$
f^{\prime \prime \prime}-R\left(f^{\prime 2}-f f^{\prime \prime}\right)-M^{2} f^{\prime}=A
$$

where $A$ is a constant. For small values of $R$, a regular perturbation scheme can be developed by expanding $f$ and $A$ in ascending powers of $R$ as

$$
f=\sum_{n=0} R^{n} f_{n}, \quad A=\sum_{n=0} R^{n} A_{n} .
$$

The coefficients of higher powers of $R(n \geq 2)$ have negligible contribution; thus we neglect them. By this method we can obtain, theoretically, the approximate solution.

Substituting (10) in (9) and equating like powers of $R$, we have

$$
f_{0}^{\prime \prime \prime}-M^{2} f_{0}^{\prime}=A_{0}, \quad f_{1}^{\prime \prime \prime}-M^{2} f_{1}^{\prime}=A_{1}+\left(f_{0}^{\prime 2}-f_{0} f_{0}^{\prime \prime}\right),
$$

etc. The corresponding boundary conditions are

$$
\begin{array}{llll}
f_{0}=\lambda, & f_{0}^{\prime}=0, & f_{n}=f_{n}^{\prime}=0 & \text { for all } n>0 \text { at } \eta=+1, \\
f_{0}=0, & f_{0}^{\prime}=1, & f_{n}=f_{n}^{\prime}=0 & \text { for all } n>0 \text { at } \eta=-1,
\end{array}
$$

where $\lambda=\left(v_{0} / c h\right)$. 


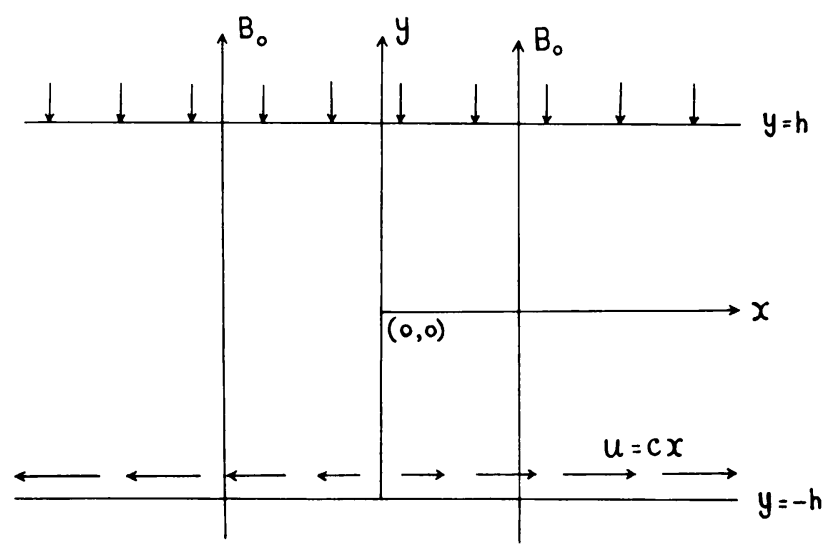

FiG. 1. Geometry of the problem.

Eqs. (11) are solved subject to the boundary conditions (12). The expression for $f_{0}$ is

$$
\begin{aligned}
f_{0}= & {[(\lambda M \cosh M-\sinh M) \eta+(1-\lambda) \sinh M \eta] / 2(M \cosh M-\sinh M) } \\
& +[(\lambda M \sinh M+\cosh M)-\cosh M \eta] / 2 M \sinh M .
\end{aligned}
$$

The expression for $f_{1}$, being lengthy, is not shown here. Figs. 2 and 3 show the effects of $\lambda$ and $M$ on the primary flow velocity and the transverse velocity, respectively. In calculating the results, we consider up to second-order approximations. When $M$ is constant, the effect of $\lambda$ on $f^{\prime}$ is maximum almost at the mid-distance from the plates. The primary flow velocity increases with $\lambda$. When $\lambda$ is constant, the effect of the Lorentz force on the primary flow is to increase it near the upper plate and to decrease it near the lower plate. Fig. 3. shows that the Lorentz force decreases the transverse velocity, i.e., $f$, with the

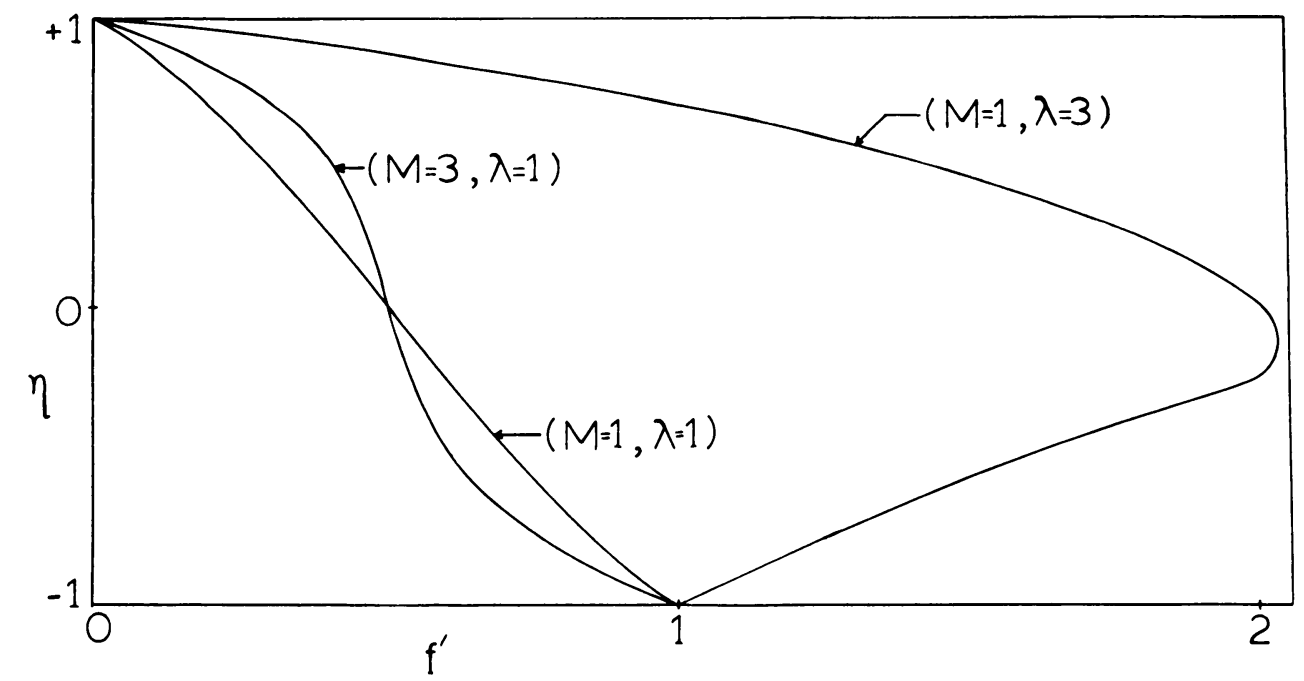

FIG. 2. Variation of $f^{\prime}(\eta)$ with the parameters $\lambda, M$. 


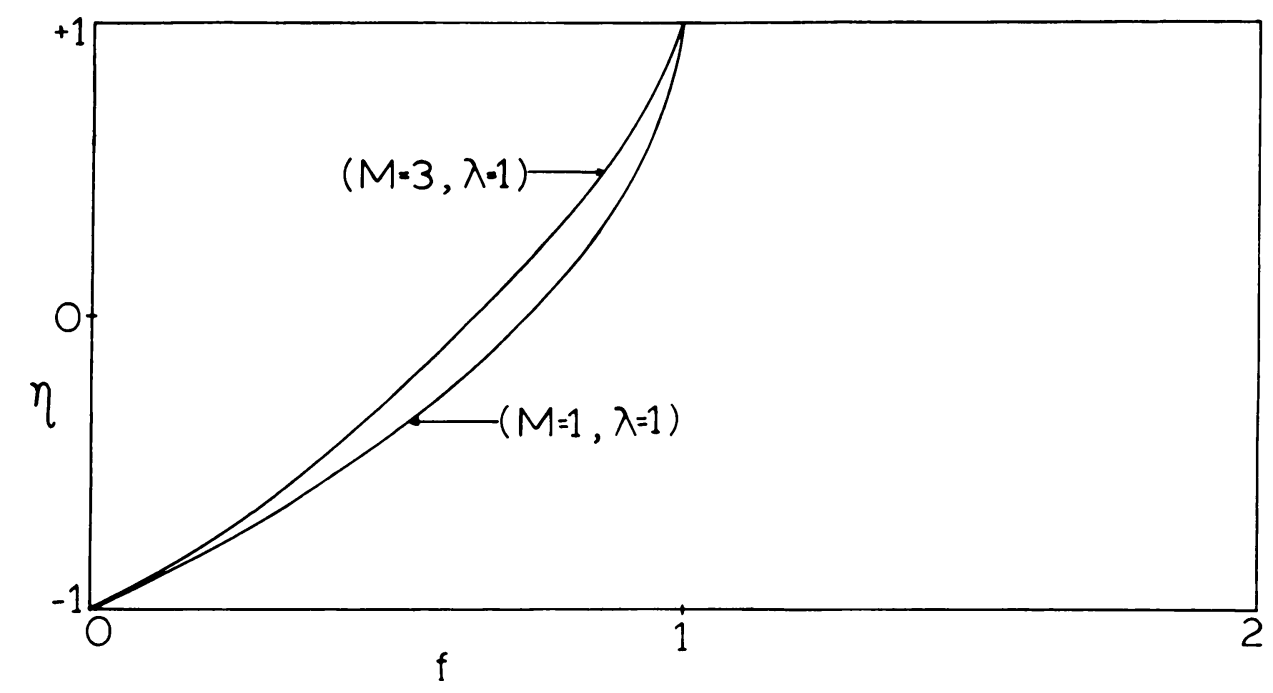

Fig. 3. Variation of $f(\eta)$ with the parameters $\lambda, M$.

increase of the magnetic field strength when $\lambda$ is constant, but the value of $f$ near the lower plate increases with the increase of $\lambda$ when $M$ is constant.

3. Heat transfer. The energy equation is

$$
\begin{gathered}
\rho c_{p}\left[u \frac{\partial T}{\partial x}+v \frac{\partial T}{\partial y}\right]=k\left[\frac{\partial^{2} T}{\partial x^{2}}+\frac{\partial^{2} T}{\partial y^{2}}\right]+\phi+\frac{\mathbf{J}^{2}}{\sigma} \\
\phi=2 \rho v\left[\left(\frac{\partial u}{\partial x}\right)^{2}+\left(\frac{\partial v}{\partial y}\right)^{2}+\frac{1}{2}\left(\frac{\partial u}{\partial y}+\frac{\partial v}{\partial x}\right)^{2}\right]
\end{gathered}
$$

where

is the viscous dissipation term and the last term is due to ohmic dissipation.

We consider here two different cases: (i) when the plates are maintained at different temperatures; (ii) when the lower plate is adiabatic and the upper plate is maintained at a constant temperature.

Case 1. when the plates are at different temperature. The boundary conditions are

$$
T=T_{1} \text { at } y=+h, \quad T=T_{0} \text { at } y=-h \quad\left(T_{0}<T_{1}\right) .
$$

Using the expression for temperature $T$

$$
T=T_{0}+\left(c^{2} h^{2} / R c_{p}\right)\left[\phi(\eta)+\frac{x^{2}}{h^{2}} \psi(\eta)\right],
$$

Eq. (14) reduces to (comparing coefficients of and the terms independent of $x$ )

$$
\begin{gathered}
\psi^{\prime \prime}=P_{\gamma} R\left[\left(2 f^{\prime} \psi-f \psi^{\prime}\right)-\left(f^{\prime \prime 2}+M^{2} f^{\prime 2}\right)\right], \\
\phi^{\prime \prime}+2 \psi=-P_{\gamma} R\left[4 f^{\prime 2}+f \phi^{\prime}\right],
\end{gathered}
$$

where $P_{\gamma}=\left(\rho v c_{p} / k\right)$.

We expand $\phi$ and $\psi$ in powers of $R$ as

$$
\phi=\sum_{n=0} R^{n} \phi_{n}, \quad \psi=\sum_{n=0} R^{n} \psi_{n} .
$$


Using (18) in Eq. (17) and comparing coefficients of like powers of $R$, we have

$$
\begin{gathered}
\psi_{0}^{\prime \prime}=0, \quad \phi_{0}^{\prime \prime}+2 \psi_{0}=0, \\
\psi_{1}^{\prime \prime}=P_{\gamma}\left[2 f_{0}^{\prime} \psi_{0}-f_{0} \psi_{0}^{\prime}-f_{0}^{\prime 2}-M^{2} f_{0}^{\prime 2}\right], \\
\phi_{1}^{\prime \prime}+2 \psi_{1}=-P_{\gamma}\left[4 f_{0}^{\prime 2}+f_{0} \phi_{0}^{\prime}\right], \quad \text { etc. }
\end{gathered}
$$

The boundary conditions (15) reduce to

$$
\begin{gathered}
\phi_{n}=0=\psi_{n} \text { for all } n \text { at } \eta=+1, \\
\phi_{0}=s, \quad \psi_{0}=0, \quad \phi_{n}=\psi_{n}=0 \text { for all } n>0 \text { at } \eta=-1,
\end{gathered}
$$

where

$$
s=\left(T_{1}-T_{0}\right) R c_{p} / c^{2} h^{2} .
$$

Eqs. (19) are solved subject to the boundary conditions (20):

$$
\phi_{0}=\frac{s}{2}(1+\eta), \quad \psi_{0}=0 .
$$

The expressions for $\phi_{1}$ and $\psi_{1}$ are not shown here (in calculating the results, we consider up to second-order approximations).

The non-dimensional expression for temperature is obtained from (16) as

$$
\begin{aligned}
\left(T-T_{0}\right) /\left(T_{1}-T_{0}\right) & =\left[\phi_{0}+R \phi_{1}+\frac{x^{2}}{h^{2}}\left(\psi_{0}+R \psi_{1}\right)\right] / s \\
& =(1+\eta) / 2+E \phi_{1}+E X^{2} \psi_{1}
\end{aligned}
$$

where $E=R / s$ and $X=x / h$. For a moderate distance from $y=$ axis, the second term is negligible in comparison to other terms [1-3]. Hence (23) reduces to

$$
\left(T-T_{0}\right) /\left(T_{1}-T_{0}\right)=(1+\eta) / 2+E X^{2} \psi_{1} .
$$

Figure 4 shows the temperature distribution for $M=1,3$ and $\lambda=1,3$ when $P_{\gamma}$ $E X^{2}=20$. The temperature at any point in the fluid increases with the increase of both $\lambda$ and $M$.

Case 2: When the lower plate is adiabatic. In this case, the boundary conditions are

$$
T=T_{1} \text { at } y=h, \quad \partial T / \partial y=0 \text { at } y=-h .
$$

Instead of (16), we use the expression for temperature

$$
T=\left(c^{2} h^{2} / R c_{p}\right)\left[\phi(\eta)+\frac{x^{2}}{h^{2}} \psi(\eta)\right] .
$$

Proceeding as in case 1, we obtain, again, Eq. (19).

The boundary conditions become

$$
\begin{aligned}
& \phi_{0}=s_{1}, \quad \psi_{0}=0, \quad \phi_{n}=\psi_{n}=0 \text { for all } n>0 \text { at } \eta=+1, \\
& \phi_{n}^{\prime}=\psi_{n}^{\prime}=0 \text { for all } n>0 \text { at } \eta=-1,
\end{aligned}
$$

where $s_{1}=\left(T_{1} R c_{p} / c^{2} h^{2}\right)$.

The expressions for $\phi_{0}$ and $\psi_{0}$ are

$$
\phi_{0}=s_{1}, \quad \psi_{0}=0 .
$$




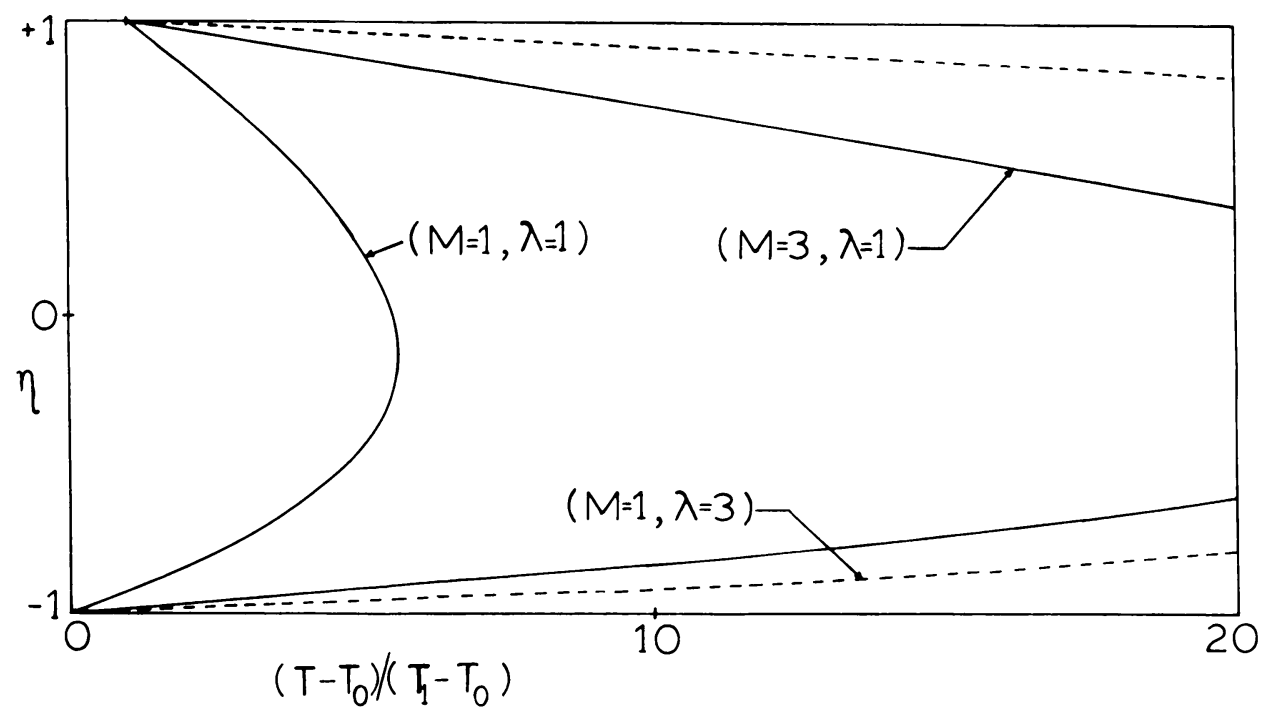

Fig. 4. Temperature distributions (plates at different temperatures).

The other expressions are not shown here. At a moderate distance from $y$-axis, the nondimensional expression for temperature is

$$
T / T_{1}=1+E X^{2} \psi_{1} .
$$

Figure 5 shows the temperature distribution for $M=1,3$ and $\lambda=1,3$ when $P_{y} E X^{2}=20$. Here also increasing values of $M$ and $\lambda$ result in increasing temperatures at any point in the fluid.

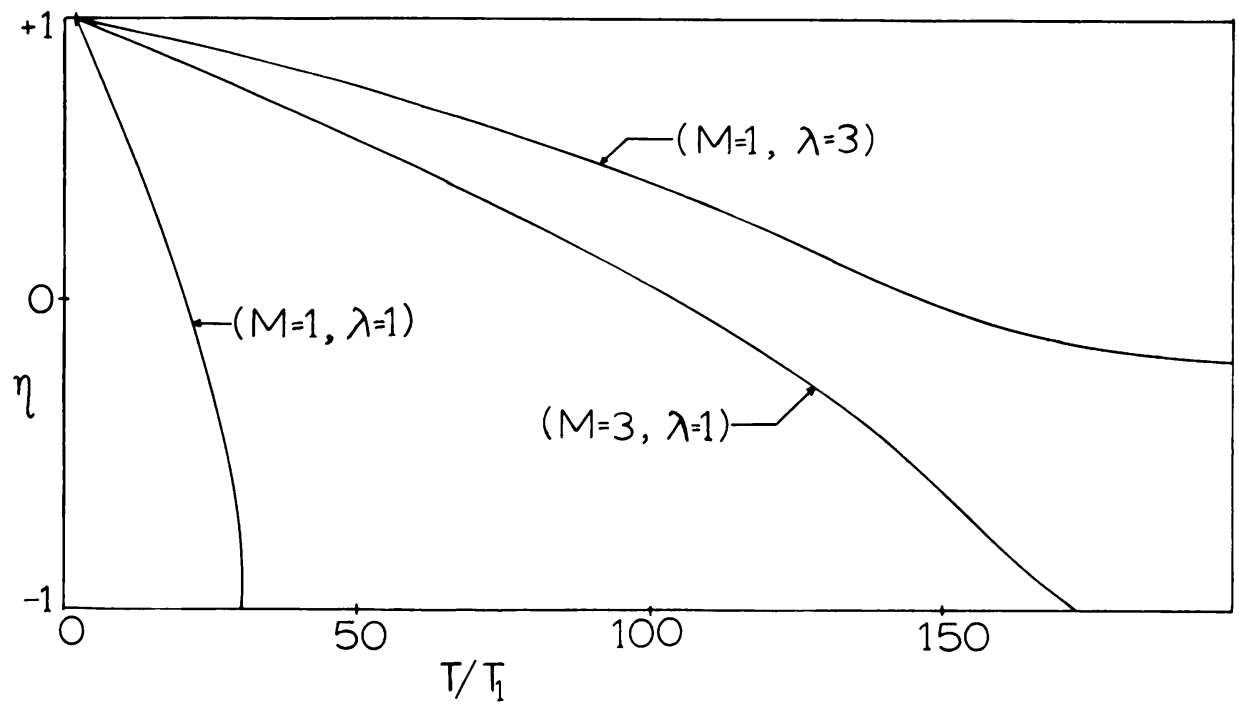

FIG. 5. Temperature distributions (adiabatic lower plate). 
4. Conclusion. $\lambda$ increases with the increase of the injection velocity and/or the decrease of the stretching velocity of the lower plate. If the stretching velocity is kept constant and the injection velocity at the upper plate is increased, the primary flow velocity increases and the maximum velocity occurs at the mid-plane of the channel (since the injection velocity has no effect on the fluid motion at the lower plate; see Eq. (4)). The same phenomenon is observed if the injection velocity is constant and the stretching velocity at the lower plate decreases. Also $F$, i.e. the transverse velocity, which vanishes at the lower plate, increases near the upper plate with increasing $\lambda$.

The imposed magnetic field is perpendicular to the primary fluid velocity $u$ and parallel to the injection velocity $V_{0}$. Since the Lorentz force acts on the primary fluid flow (this velocity is $c x$ at the lower plate and zero at the upper plate) in the opposite direction (see Eq. (1)), the increase of the magnetic field strength decreases $u$ at the lower plate and has little effect on the velocity near the upper plate.

The energy equation (14) contains both viscous dissipation and ohmic dissipation. The temperature changes due to the internal friction which causes viscous dissipation depend on the flow velocity - it is negligible for small velocities and becomes important at high velocities. Joulean or Ohmic dissipation represents the rate at which electromagnetic energy is converted into heat. As the fluid flow velocity and the electric current generated in the fluid increase with $\lambda$ and the strength of the magnetic field respectively, the fluid temperature increases with both $\lambda$ and $M$.

\section{REFERENCES}

[1] A. C. Srivastava, A1AA 2, 749 (1963)

[2] A. C. Srivastava and S. K. Sharma, J. Phys. Soc. Japan 19, 1390 (1964)

[3] A. Bharali and A. K. Borkakati, App. Sci. Res. 35, 161 (1979)

[4] B. Chandrasekhara and N. Rudraiah, App. Sci. Res. 23, 42 (1970)

[5] A. Chakrabarti and A. S. Gupta, Quart. Appl. Math. 37, 73 (1979) 